\title{
An Analysis of the Vi-Phage Typing Scheme for Salmonella typhi
}

\author{
By A. BERNSTEIN* AND E. MAUREEN J. WILSON \\ Enteric Reference Laboratory, Public Health Laboratory Service, \\ Colindale Avenue, London N.W. 9
}

(Received 17 January 1963)

\begin{abstract}
SUMMARY
The seventy-two currently recognized Vi-phage types of Salmonella typhi have been examined in order to determine, where possible, their structural formulae. The genetic nature of the changes in host range of the corresponding typing adaptations of the Vi-phage II has also been investigated.

Thirty-seven temperate phages have been isolated from the type strains and type-determining ability has been demonstrated in all but five of these phages. Several of the determining phages were found in more than one type strain and it is probable that no more than 16 different determining phages are represented in our collection. Because there are fewer phage reactions to be eliminated in cultures already possessing a restricted sensitivity spectrum to the typing adaptations of Vi-phage II, some of the determining phages ('semi-determining phages') display their full effect only in such cultures.

One determining phage, d1-C, can co-operate with others, especially phages $\mathrm{d} 8$ and $\mathrm{d} 9$, in multiply lysogenized cultures to produce an effect which is more than a summation of the known type-determining powers of the individual phages concerned. Moreover, the type-determining effects of phages d1-C, d8, and d9 in singly or doubly lysogenized cultures are influenced by the temperature of incubation.

As has been shown by earlier workers, the changes in host-range of Vi-phage II that produce the Vi-typing phages may be phenotypic (hostinduced) or genotypic (mutational) in nature, or a combination of these two types of variation. Amongst the current typing phages are represented eighteen different phenotypic modifications and forty-three different hostrange mutants. Many of these mutations seem to be related to each other but their chromosomal distribution has not been mapped.
\end{abstract}

\section{INTRODUCTION}

Vi-phage typing of Salmonella typhi has been in routine use for more than 20 years and has continued to justify the initial hopes that were placed in it as an epidemiological tool in the study of typhoid fever (Craigie \& Felix, 1947). The typing scheme in current international use contains 72 types of $S$. typhi and their diagnostic reactions with the various typing adaptations of Vi-phage II are shown in Table 1. In this paper, the expressions type, phage-type and Vi-type refer to the bacterial strains recognized by the Vi-typing phages. Similarly, Vi-phage, Vi-

* Present address: Department of Microbiology and Immunology, Marquette University School of Medicine, Milwaukee, Wisconsin, U.S.A. 
typing phage and typing phage refer to the typing preparations adapted from Vi-phage II.

In the Vi-phage-typing scheme for Salmonella typhi, the phage types of the organism and the corresponding adaptations of Vi-phage II carry the same symbol. Thus, Vi-type $\mathbf{A}$ is lysed by phage $\mathbf{A}$, type $\mathbf{B} 1$ by phage $\mathbf{B} 1$ and so on. In order to avoid confusion, Anderson (1962 $a$ ) recently adopted the practice of italicizing the symbol of the typing phage, and we shall follow this convention.

All the Vi-typing phages are descended from the original Vi-phage II of Craigie \& Yen (1938). Anderson (1962a) has analysed the genetic mechanisms which underlie the processes of Vi-phage adaptation to, and Vi-type determination in, the various phage-types of Salmonella typhi. This analysis is based on several past investigations and in particular those of Anderson \& Fraser (1955, 1956). The original paper of Anderson (1962 a) should be consulted for a fuller account and for a complete list of references, but for our present purposes we summarize it here.

It has been shown (Anderson \& Fraser, 1955, 1956) that the Vi-typing preparations fall into four genetic categories : the wild-type of Vi-phage II which is phage $\boldsymbol{A}$; preparations that have undergone only host-induced or phenotypic modification (Luria \& Human, 1952; Anderson \& Felix, 1952, 1953 $a$; Luria, 1953; Bertani \& Weigle, 1953; Weigle \& Bertani, 1953); those that are pure host-range mutants; and those that represent a combination of phenotypic and mutational changes.

The wild-type of Vi-phage II lyses only type A. This type is also sensitive to all the typing adaptations of Vi-phage II and is regarded as the most primitive Vi-type of Salmonella typhi.

Vi-type specificity in Salmonella typhi, that is the pattern of reactions with the typing adaptations of Vi-phage II, is in many instances partly dependent on lysogenicity in the types concerned (Craigie, 1946; Felix \& Anderson, 1951 ; Anderson, 1951; Anderson \& Felix, 1953b), and the phages having this function are called type-determining phages. The remainder of the phage sensitivity in these lysogenic types is controlled by their non-lysogenic ancestors. As the types can thus be regarded as consisting of two components, the non-lysogenic precursor and the determining phage, Anderson \& Fraser (1955) suggested that they could be allotted structural formulae consisting of the symbol of the non-lysogenic precursor type followed by that of the determining phage in parenthesis; thus type 29 , which is type A carrying phage $f 2$, is structurally $A(f 2)$; type $F 2$ is type $F 1$ carrying the same phage, and is structurally $\mathrm{F} 1(\mathrm{f} 2)$; type D6 is type A carrying phage d6 and is therefore $A(d 6)$; and so on. The structural formulae are useful in the study of this typing system, because they show relationships between types that may not be indicated by the symbols in routine use, and also, as will be seen, give information on the probable genetic nature of the corresponding typing adaptations of Viphage II.

Lysogenization of precursor types with determining phages has yielded types identical with those found in nature; and spontaneous loss of determining phages by naturally occurring lysogenic types has revealed the presence of the precursor types they had been postulated to possess (Anderson \& Felix, 1953 $b$; Anderson \& Fraser, 1955). These findings indicate the validity of the structural formulae and suggest the mechanism of formation of the lysogenically determined types in nature. 


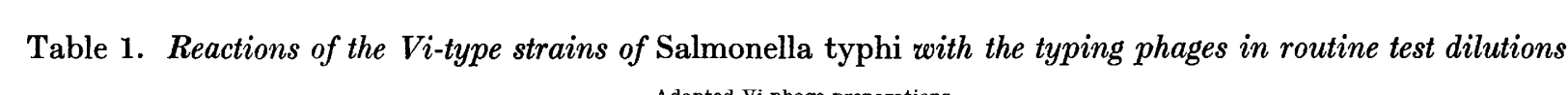

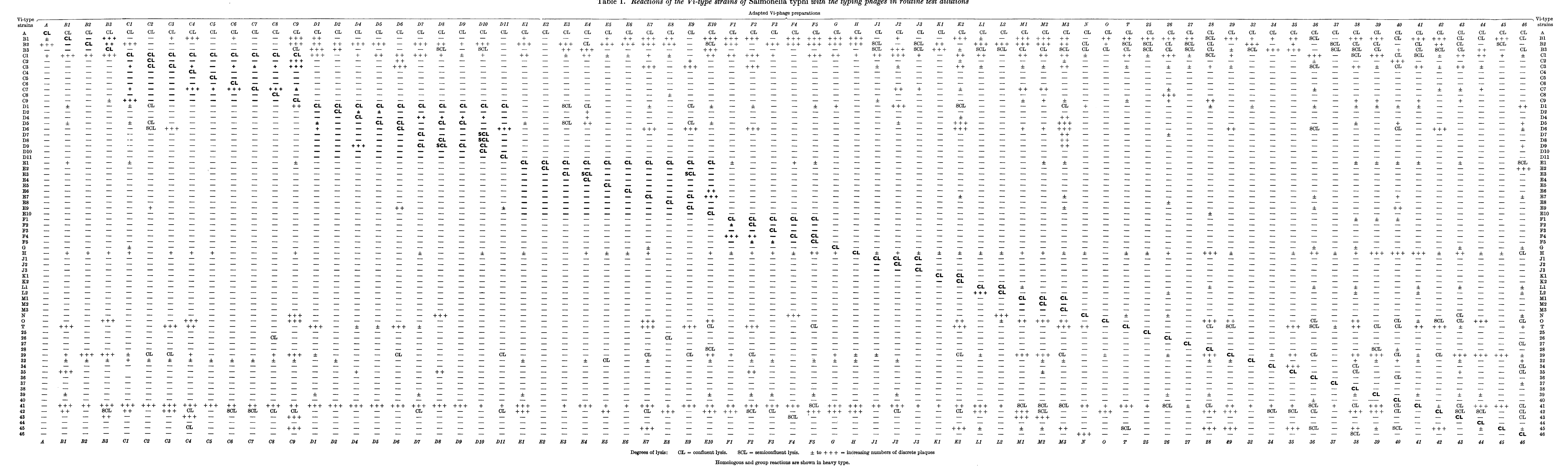


The commonest Vi-types of Salmonella typhi are A, C1 and E1. These are nonlysogenic, and it has been found that they are also the commonest precursors of lysogenically determined types. Anderson \& Fraser (1955) showed that typing preparations of Vi-phage II that had undergone a purely phenotypic change were those adapted to non-lysogenic types such as $\mathbf{A}, \mathbf{C} 1$ and $\mathrm{E} 1$. The typing phages consisting of pure host-range mutants of Vi-phage II were those adapted to lysogenically determined types whose bacterial precursors were type $\mathbf{A}$. The phages combining phenotypic with mutational change were those adapted to lysogenically determined types whose precursors were types other than A, such as C1 or E1. Certain types in which lysogenicity could not be demonstrated were lysed only by host-range mutants of Vi-phage II; others were lysed by phages in which phenotypic change was combined with mutation.

The examination of the host-range of a typing phage may suggest to which category the phage belongs. A pure host-range mutant will in general lyse type $\mathbf{A}$, the homologous type strain and possibly a few other types sensitive to the mutation. Its host-range will be unaltered after growth in type $\mathbf{A}$. The pure host-induced modification will almost always attack only type $\mathbf{A}$ and the type strain to which it has been adapted. After one cycle of growth in type $\mathbf{A}$ it will retain only the ability to lyse type A, that is, it will have reverted to phage $A$. A phage which is a phenotypic modification of a host-range mutant will lyse type $\mathrm{A}$, the homologous type strain, and any type sensitive to the basic mutation. It will also lyse any precursor types such as $\mathrm{C1}$ or E1, which happen to be represented in its homologous type. Growth of such a phage in type A will lead to loss of the ability to attack both the homologous type strain and the precursor type, but not of that to lyse type $A$ and the cultures sensitive to the basic mutation. If no cultures exist which are sensitive to the basic mutation it may be difficult to distinguish this type of phage from a pure phenotypically modified one. However, when the precursor is known this distinction may be made by regrowing the phage which has been propagated on type $\mathbf{A}$ in the precursor type. Under these circumstances a phage which was originally a pure host-induced modification will lyse only type $\mathbf{A}$ and the precursor type, whereas a phage which was a phenotypic modification of a host-range mutant will have regained all its original potentialities. It may also be possible in some cases to lysogenize type $\mathbf{A}$ with a determining phage isolated from the strain to which the Vi-phage had been adapted and so to produce a culture which is resistant to phage $A$ but sensitive to both the original phage and that resulting from its growth in type A. This shows that the phage grown on type A has not reverted to phage $\dot{A}$ but must be a host-range mutant of Vi-phage II.

Types E1, E2, E5, and E8 and their corresponding typing phages provide a convenient illustration of the way in which some of the combinations of phenotypic and mutational changes in host-range of Vi-phage II may be characterized. These four types are all members of the $\mathbf{E}$ group of the Vi-typing scheme (Table 1 ) but only E8 is known to be lysogenic. Type E1 is one of the common precursor types and typing phage $E 1$ has been shown to be a pure phenotypic modification of Vi-phage II (Anderson \& Felix, 1952, 1953a; Anderson \& Fraser, 1955, 1956). Growth of phage $E 1$ on type A produces a phage indistinguishable from phage $\boldsymbol{A}$.

Type E8 carries a phage identical with phage $26^{\prime}$, which is the determining phage of types 26 and C8. The typing phage $E 8$ lyses types A, E1, and 26 in addition to 
E8. After growth in type $\mathrm{A}(\rightarrow E 8 / \mathrm{A})$ this phage resembles phage 26 and lyses only types $A$ and 26. Fractional symbols such as $X / Y$ are used to designate the phage produced by the growth of phage $X$ in type $Y$. The phage resulting from the further propagation of $X / Y$ in type $Z$ would be designated $X / Y / Z$, and so on. Growth of phage $E 8 / \mathrm{A}$ on type E1, or of phage 26 on type E1, produces a phage indistinguishable from phage $E 8$. The host-range of $E 8$ therefore appears to be due to two changes. One is mutational and is identical with the mutation represented in phage 26 whereas the other is phenotypic and is the same as that in phage $E 1$.

Typing phages $E 2$ and $E 5$ both lyse types $A$ and $E 1$ in addition to their respective type strains. Phage $E 5$ also lyses type $\mathbf{3 2}$ for reasons at present unknown. After growth on type $\mathbf{A}(\rightarrow E 2 / \mathbf{A}$ and $E 5 / A$ respectively) both phages lyse only type $\mathbf{A}$ and thus appear to have reverted to phage $A$. However, growth of phage $E 5 / \mathrm{A}$ in type E1 gives phage $E 1$, whereas growth of E2/A in type E1 produces phage E2. From this it follows that $E 2 / \mathrm{A}$ is not identical with phage $A$, but must represent a hostrange mutant of Vi-phage II for which there is at present no indicator strain available other than type A. Phage E2 is, therefore, a phenotypically modified host-range mutant, the phenotypic change being identical with that in phage $E 1$. Phage E5, on the other hand, represents a pure phenotypic modification of host-range of $\mathrm{Vi}$ phage II, although the lytic spectrum of phage $E 5$ is rather wider than is usual with this class of adaptation.

Anderson \& Fraser (1955) investigated the various Vi-types of the typing scheme as it then existed and it is from their observations that the principles given above have been developed. At the time of their survey the number of phage types was thirty-three. The number of types has now increased to seventy-two and it was considered worth while, therefore, to record the results of the investigation of the many new types which have been discovered since 1955. In the course of the survey some new determining phages have been isolated and new facts discovered about some of those previously known.

Our techniques, which have developed from those of Anderson \& Fraser (1955), are based on the principles presented above and are designed to elicit as much information as possible about the type strains and their typing phages.

\section{METHODS}

Media. The liquid medium was Bacto dehydrated nutrient broth (Difco Laboratories) used at a strength of $2 \%(\mathrm{w} / \mathrm{v})$ in distilled water with $0.85 \%(\mathrm{w} / \mathrm{v})$ added $\mathrm{NaCl}$.

For solid medium New Zealand powdered agar was added to a final concentration of $1.3 \%(\mathrm{w} / \mathrm{v})$. The surface layer of agar layer plates contained only $0 \cdot 4 \%(\mathrm{w} / \mathrm{v})$ agar.

Phage-typing. The methods given by Anderson \& Williams (1956) were used for the phage-typing of all cultures. Unless otherwise stated, the phages were used at their Routine Test Dilution (RTD), that is, the highest dilution producing confluent lysis of the homologous type strain. The temperature of incubation was $38 \cdot 5^{\circ}$ unless specifically mentioned.

Phage titrations. All phage preparations were titrated by the spot technique. For more accurate assays agar layer titrations were performed (Adams, 1959).

'Phage sterilization'. Phage preparations were rendered bacteriologically sterile by one of three techniques. All Vi-phage II 'adaptations' were heated in a water 
bath at $57^{\circ}$ for $\mathbf{4 0} \mathrm{min}$. Some temperate phages were also treated in the same way. In many cases, however, the temperate phages were found to be labile at this temperature; they were then sterilized either by treatment with toluene (Anderson $\&$ Felix, 1953 $b$ ) or by filtration through sintered glass (a.p.d. 1.2-1.4 $\mu$ ).

Determination of lysogeny. Our usual method for the detection of lysogeny was to inoculate $c .10^{8}$ organisms each of type $\mathrm{A}$ and of the strain under examination jointly into $20 \mathrm{ml}$. of pre-warmed broth. This mixed culture was incubated at $37^{\circ}$ with agitation. The supernatant of the culture was examined after 5 and $17 \mathrm{hr}$. incubation for evidence of phage activity. The indicator strains used for this purpose were type $A$, the strain under survey, and one or two other selected types. The examination was made by the usual spotting technique and plates were incubated at $36 \cdot 5^{\circ}$ and $38 \cdot 5^{\circ}$ overnight.

If the preparation showed evidence of phage activity, a small portion (c. $2 \mathrm{ml}$.) of the lytic supernatant was heat-sterilized and the original and heated preparations were titrated in parallel on type $\mathbf{A}$ and any other sensitive indicators. A phage which was found to be heat-labile was sterilized by toluene treatment or filtration.

Heat-stable phages were purified by cutting several single plaques from the titration on type A. Care was taken to remove as little of the surrounding culture as possible and the plaque was emulsified in $3 \mathrm{ml}$. of broth which was then heatsterilized. This preparation was again titrated on type $A$ and similar single-plaque selection was made from at least one more titration. In cutting plaques from the final titration, some of the surrounding culture was included in the pick and the whole was emulsified in $20 \mathrm{ml}$. pre-warmed broth. The inoculated broths were incubated at $37^{\circ}$ for $5 \mathrm{hr}$. and the supernatants of these final preparations were sterilized by heat. Purification and propagation of heat-labile phages were essentially the same except that sterilization was by either filtration or the use of toluene. Some cultures proved to be doubly lysogenic, and in such cases it was usually found that one of the carried phages was stable and the other labile at $57^{\circ}$. The phages isolated from such cultures appeared to be partially heat-sensitive, because of the elimination of the heat-labile component at $57^{\circ}$. This provided an easy method of obtaining pure lines of the heat-stable phage. Titrations of filtered preparations of such mixed phages were carefully explored for diversity of plaque types, in order to identify those of the heat-labile phage.

During the isolation of temperate phages, the various preparations were carefully examined at each stage of plaque selection for evidence of heterogeneity with respect to both plaque morphology and host-range. If such heterogeneity was found, single-plaque selection was made of representatives of each type of variation.

With some temperate phages 'induction' of the lysogenic culture by ultraviolet light was found to improve the phage yield.

Purification and propagation of typing phages. 'The phage concerned was titrated on a suitable host strain and single plaques were emulsified in $3 \mathrm{ml}$. broth. If the titration showed heterogeneity of plaque morphology, representatives of each plaque type were chosen. The broth preparations were sterilized by heat and titrated on the same host strain and on any other strains which appeared to be suitable indicators of host-range. Further single plaque selections were made and titrated as before. Finally, single plaques representing all of the different plaque and host-range variants were picked and emulsified in $20 \mathrm{ml}$. pre-warmed broth which was in- 
cubated at $37^{\circ}$ with agitation until lysis occurred. If lysis was not apparent the incubation was interrupted at $5 \mathrm{hr}$. The suspensions were centrifuged at $1500 \mathrm{~g}$ and sterilized by heating to $57^{\circ}$ for $40 \mathrm{~min}$.

In order to obtain higher titre lysates propagation was also carried out by the agar-layer technique (Swanstrom \& Adams, 1951) after initial single-plaque purification.

Adaptation of Vi-phage II. The phage to be adapted was titrated on the selected strain and single plaques, if any, were transferred to $3 \mathrm{ml}$. broth, sterilized by heat, and examined for change in host-range. Further single-plaque selection and final propagation were performed as in the preceding section. In this paper the term 'de-adapted' will be used to refer to any phage, other than $A$, which has been grown on type A. Propagations of a phage on types other than its homologous type will be referred to as 'adaptations'.

Characterization of host-range of typing phages. The host-range of the Vi-phages was usually ascertained at the R'TD. This dilution is chosen so as to minimize the number of cross-reactions on strains other than the homologous type. The statement that phage $X$ does not lyse type $\mathbf{Y}$ may only be true, therefore, at the RTD or higher dilutions.

The RTD of a given phage is influenced by the absolute content of plaqueforming particles in the preparation, by the size of the plaques formed by the phage on its type strain, and by the efficiency of plating (EOP) of the phage. The interaction of these factors is discussed later.

The EOP of a phage is a measure of its plaque-forming ability on a given strain relative to some other, usually higher, estimate of phage content (Adams, 1959). For the purposes of this paper, the EOP of a phage on type $\mathrm{X}$ is the ratio of plaque count on type $\mathbf{X}$ to that on type $A$. The maximum possible value of the EOP is 1 .

Lysogenization. Sterilized, undiluted preparations of the various single-plaque lines of the phage concerned were spotted on a lawn of the strain to be lysogenized. After overnight incubation the secondary growth from the central lysed area was inoculated into $20 \mathrm{ml}$. pre-warmed broth, which was incubated overnight. Serial passages were then made by inoculating $1 \mathrm{ml}$. of culture into $20 \mathrm{ml}$. of fresh broth. This was continued for several passages. Subcultures of each passage were made on Dorset's egg medium and retained for reference purposes. Each stage was examined to detect any loss of sensitivity to the lysogenizing phage. Tests were also made of the ability of the cultures to release the temperate phage used for lysogenization and for any change in reaction of the host strain to the typing adaptations of Viphage II. For the latter test a restricted range of typing phages was usually sufficient. When these tests suggested that lysogenization had occurred, the culture was plated and several single colonies picked and tested. Serial single-colony selection was carried out until the lines chosen appeared to be stable and homogeneous. The final single-colony lines were tested with all the typing phages in RTD.

In some cases, especially where plaques were minute, secondary growth could not be harvested from surface phage titration. In such cases agar-layer titrations were performed with phage concentrations sufficiently high to yield confluent lysis. Lysogenized secondary growth was usually easy to obtain from these titrations. 


\section{RESULTS}

The investigation of various types, including new types, was conducted according to a uniform plan. This derives from the principles outlined earlier and may conveniently be divided into three main stages.

Stage $I$. The type strain was phage typed in the routine way using all the existing typing phages, and, in addition, for the new types, the homologous adapted preparations. The host-range of the typing phage was determined, using all the accepted phage types and the new type.

Stage $I I$. Phage $A$ was titrated on the type strain and attempts were made to propagate any plaques detected. If no reaction was detected with phage $A$, similar attempts were made with other known typing phages, especially any which had shown evidence of an ability to lyse the new type.

When it was possible to isolate a temperate phage from the new type, this phage was propagated and several clonal lines were used to lysogenize a variety of cultures. These always included type A, one or more of the common precursor types (such as $\mathrm{C} 1, \mathrm{E} 1, \mathrm{~F} 1$ ), and also any possible precursor types which showed evidence of sensitivity to the new typing phage.

The new typing phage was titrated on the newly lysogenized strains and attempts were made to adapt it to them. It was also de-adapted by growth on type A and then re-adapted to several of the precursor strains.

Stage III. A full cross-test was performed using, in addition to all the phages mentioned in stage $I$, all the adapted preparations used in routine typing, together with other typing phages obtained in stage II. The de-adapted new phage and its various re-adaptations were also included, as were the preparations of any temperate phages isolated from the new type. These phages were tested against all the type strains, including the new type, plus the lysogenized cultures prepared in stage II.

It is usually possible from the results of such a test to deduce the genetic nature of the typing phage, and often also the structural formula of the type strain and the type-determining ability of any temperate phage isolated from it. Possible crossrelationships between the temperate phage and previously known determining phages may also be detected. On the basis of these tests a decision can be made as to which of several single-plaque lines of the typing phage is most suitable for use in routine typing.

Table 2 summarizes all the pertinent information on the structure of the Vitypes and on the genetic nature of the typing phages. We have included for completeness types previously examined by other workers, whether or not we have reinvestigated them.

Our system of nomenclature for temperate phages, for lysogenized strains, and for phage adaptations follows that of Felix \& Anderson (1951), Anderson \& Felix $(1953 a, b)$ and Anderson \& Fraser (1955). We have been conservative in the naming of new determining phages; for example the phages isolated from types $26, \mathrm{C} 8$ and E8 are indistinguishable from each other and we prefer to call them all phage $26^{\prime}$ (the phage first isolated from type 26). We believe this to be of use in indicating the relationship between various types, even when full identity of the determining phages has not been established. 
Table 2. Summarized information on the Vi-types of Salmonella typhi and their homologous typing phages

\begin{tabular}{|c|c|c|c|c|c|}
\hline \multirow[b]{2}{*}{ Vi-type } & \multicolumn{2}{|c|}{ Type strain } & \multicolumn{2}{|c|}{$\begin{array}{l}\text { Nature of change in } \\
\text { typing phage }\end{array}$} & \multirow[b]{2}{*}{ References* } \\
\hline & $\begin{array}{l}\text { Phages } \\
\text { carried }\end{array}$ & $\begin{array}{c}\text { StructuraI\$ } \\
\text { formula }\end{array}$ & Phenotypic & $\overbrace{\text { Genotypic }}$ & \\
\hline $\mathbf{A}$ & & $\mathbf{A}$ & & & \\
\hline B1 & & & & $\mathbf{B 1}$ & 8 \\
\hline B2 & $\mathbf{b} 2 \dagger$ & & & B2 & 10 \\
\hline B3 & b3 & A(b3) & & B3 & $7,9,10$ \\
\hline C1 & & C1 & $\mathrm{Cl}$ & & 6,8 \\
\hline C2 & d6 & C1 (d6) & C1 & D6 & $2,4,8,10$ \\
\hline C3 & $\mathbf{f 2}$ & $\mathrm{Cl}(\mathrm{f} 2)$ & C1 & 29 & $2,4,7,10$ \\
\hline $\mathrm{C} 4$ & & & $\mathrm{C} 1$ & $\mathrm{C} 4$ & 5 \\
\hline C5 & $\mathbf{c 5} \dagger$ & & C1 & C5 & $\mathbf{5}$ \\
\hline C6 & c6† & & $\mathrm{Cl}$ & C6 & $\mathbf{5}$ \\
\hline $\mathrm{Cr}$ & & & C1 & $\mathrm{C7}$ & 5 \\
\hline C8 & $\mathbf{2 6}^{\prime}$ & $\mathrm{Cl}\left(26^{\prime}\right)$ & C1 & 26 & 4,5 \\
\hline C9 & $\mathbf{c 9} \ddagger$ & $\mathrm{Cl}(\mathrm{c} 9)$ & C1 & B3 & 4,5 \\
\hline D1 & d1-C or d1-E & $A(d 1-C)$ or $A($ & dl-E) & D1 & $2,4,7,8,9,10,11,12$ \\
\hline D2 & & & D2 & D1 & 8 \\
\hline$\overline{\mathrm{D} 4}$ & d1-C & $?(\mathrm{~d} 1-\mathrm{C})$ & D4 & D1 & $2,7,8,9,11,12$ \\
\hline D5 & & & & D5 & $6,8,12$ \\
\hline D6 & d6 & $A(d 6)$ & & D6 & $2,4,6,7,8,11,12$ \\
\hline D7 & d1-C & $?(\mathrm{~d} 1-\mathrm{C})$ & & D7 & $5,9,14$ \\
\hline D8 & $\mathrm{d} 1-\mathrm{C}+\mathrm{d} 8 \ddagger$ & $A(d 1-C)(d 8)$ & & D8 & $4,5,9,14$ \\
\hline D9 & $\mathrm{d1}-\mathrm{C}+\mathrm{d} 9+$ & $A(d 1-C)(d 9)$ & & D9 & $4,9,14$ \\
\hline D10 & $\mathrm{d} 1-\mathrm{C}+\mathrm{d} 8 \ddagger$ & $?(\mathrm{~d} 1-\mathrm{C})(\mathrm{d} 8)$ & & D10 & $4,9,14$ \\
\hline D11 & d6 & $?(\mathrm{~d} 6)$ & & D11 & \\
\hline E1 & & E1 & E1 & & 6,8 \\
\hline E2 & & & E1 & $\mathrm{Cr}$ & 8 \\
\hline E3 & & & E1 & E3 & 5,16 \\
\hline E4 & & & E1 & E4 & 5,16 \\
\hline E5 & & E5 & E5 & & 5 \\
\hline E6 & & & E1 & E6 & $\mathbf{5}$ \\
\hline E7 & f2 & E1(f2) & E1 & 29 & $4,5,7,11,15$ \\
\hline E8 & $\mathbf{2 6}^{\prime}$ & $\mathrm{E} 1\left(26^{\prime}\right)$ & E1 & 26 & $2,4,5,8,12$ \\
\hline E9 & d6 & E1 (d6) & E1 & D6 & 3,5 \\
\hline E10 & t-def? & E1 (t-def) & E1 & 28 & \\
\hline F1 & & F1 & F1 & & 1,6 \\
\hline F2 & f2 & F1(f2) & F1 & 29 & $1,2,4,7,8,12$ \\
\hline F3 & f3t & & F1 & F3 & \\
\hline F4 & & & F1 & 43 & \\
\hline F5 & $\mathbf{t}$ & F1 $(t)$ & F1 & $\mathbf{T}$ & \\
\hline G & & G & $\mathbf{G}$ & & 8 \\
\hline $\mathbf{H}$ & & $\mathbf{H}$ & $\mathbf{H}$ & & 8 \\
\hline J1 & & $\mathbf{J 1}$ & $\mathbf{J 1}$ & & 8 \\
\hline $\mathbf{J 2}$ & j2 & $\mathbf{J 1}(\mathbf{j 2})$ & J1 & J2 & 4 \\
\hline J3 & & & J1 & J3 & \\
\hline K1 & $\mathbf{k} \dagger$ & K1 & & K1 & $7,8,11,12$ \\
\hline K2 & d6 & K1 (d6) & & K2 & \\
\hline L1 & & L1 & $\mathbf{L 1}$ & & 6,8 \\
\hline L2 & & & L1 & L2 & 8 \\
\hline$\overline{\mathrm{MI}}$ & & M1 & M1 & & 8,13 \\
\hline M2 & & & M1 & M2 & 13 \\
\hline M3 & d6 & M1 (d6) & M1 & D6 & 13 \\
\hline $\mathbf{N}$ & & & & $\mathbf{N}$ & 6,8 \\
\hline $\mathrm{O}$ & & & & $\mathbf{O}$ & 6,8 \\
\hline $\mathbf{T}$ & $\mathbf{t}$ & $A(t)$ & & $\mathbf{T}$ & $2,4,6,7,8,11,12$ \\
\hline 25 & $\mathbf{2 5}^{\prime}$ & $\mathbf{A}\left(25^{\prime}\right)$ & & 25 & $2,4,7,8,11,12$ \\
\hline
\end{tabular}


Table 2 (cont.)

\begin{tabular}{|c|c|c|c|c|c|}
\hline \multirow[b]{2}{*}{ Vi-type } & \multicolumn{2}{|c|}{ Type strain } & \multicolumn{2}{|c|}{$\begin{array}{l}\text { Nature of change in } \\
\text { typing phage }\end{array}$} & \multirow[b]{2}{*}{ References* } \\
\hline & $\begin{array}{l}\text { Phages } \\
\text { carried }\end{array}$ & $\begin{array}{c}\text { Structural\$ } \\
\text { formula }\end{array}$ & Phenotypic & Genotypic & \\
\hline 26 & $26^{\prime}$ & $\mathbf{A}\left(26^{\prime}\right)$ & & 26 & $2,4,7,8,11,12$ \\
\hline 27 & & & 27 & & 8 \\
\hline 28 & $t-\operatorname{def}+28^{\prime}+$ & $A(t-d e f)\left(28^{\prime}\right)$ & & 28 & $7,8,11,12$ \\
\hline 29 & f2 & $A(f 2)$ & & 29 & $7,8,11,12,16$ \\
\hline 32 & & & 32 & & 8 \\
\hline $\mathbf{3 4}$ & & & & 34 & 5 \\
\hline $\mathbf{3 5}$ & & & & 35 & $\mathbf{5}$ \\
\hline 36 & $36^{\prime} \dagger$ & & & 36 & 5 \\
\hline 37 & & & 37 & & 5 \\
\hline 38 & & & & 38 & $\mathbf{5}$ \\
\hline 39 & & & 39 & $\mathbf{T}$ & $\mathbf{5}$ \\
\hline 40 & & & 40 & 36 & $\mathbf{5}$ \\
\hline 41 & & & 41 & & 5 \\
\hline 42 & & & & 42 & 5 \\
\hline 43 & $43^{\prime} \div$ & $?\left(43^{\prime}\right)$ & & 43 & \\
\hline 44 & & & & 44 & \\
\hline 45 & & & & 45 & \\
\hline 46 & & & & 46 & \\
\hline
\end{tabular}

* References: 1, Anderson (1951); 2, Anderson (1955); 3, Anderson (1956); 4, Anderson (1959); 5, Anderson (1962b); 6, Anderson \& Felix (1952); 7, Anderson \& Felix (1953b); 8, Anderson \& Fraser (1955); 9, Bernstein (1963); 10, Craigie (1946); 11, Felix \& Anderson (1951); 12, Ferguson, Juenker \& Ferguson (1955); 13, Nicolle, Hamon \& Diverneau (1962); 14, Read \& Ferguson (1961); 15, Scholtens (1955); 16, Scholtens (1956).

$\dagger$ Phages which apparently possess no type-determining power.

$\ddagger$ Semi-determining phages (see text).

$\$$ The structural formulae have been confirmed in most cases by the synthesis of the type concerned. Where the precursor of a phage-determined type is not known, it is indicated by the symbol '?'.

For many of the types nothing can be usefully added to the data recorded in Table 2. There are, however, some types of which a more extensive account of results is necessary and these are considered in detail below. They have been collected into groups, usually on the basis of the determining phages they carry. Some have been grouped because of cross-relationships of the typing phages. Neither grouping necessarily bears any relationship to the practical groupings of the typing scheme.

$D 1$ group. We include in this group those cultures lysogenic for phage d1, that is, types D1, D4, D7, D8, D9 and D10. Other members of typing group 'D' are discussed elsewhere. All the typing phages of the group are pure host-range mutants, except phage $D 4$, which is a phenotypically modified mutant.

The precursors of types D1, D8, and D9 are all type $A$ and it has been shown by Read \& Ferguson (1961) that the differences in the phage sensitivities of these strains are due to the fact that two of them are doubly lysogenic. Read \& Ferguson gave the structural formulae of types D8, D9, and D10 as $\mathbf{A}(\mathrm{d} 8 \alpha)(\mathrm{d} 8 \beta), \mathbf{A}(\mathrm{d} 9 \alpha)$ $(\mathrm{d} 9 \beta)$, and ? $(\mathrm{d} 10 \alpha)(\mathrm{d} 10 \beta)$ respectively. The various ' $\alpha$ ' phages appeared to have identical properties and resembled phage d1. Read \& Ferguson also claimed the ' $\beta$ ' phages to be different from any others previously described. Phage $\mathrm{d} 8 \beta$ appeared to be identical with phage $\mathrm{d} 10 \beta$ but $\mathrm{d} 9 \beta$ was completely distinct. 
Our investigations of these types have produced results similar to those of Read $\&$ Ferguson (1961). Since the three ' $\alpha$ ' phages are indistinguishable from each other and from $\mathrm{d} l$ (but see later) we prefer to give them all the same designation of $\mathrm{d} l$. By doing this the necessity for the ' $\alpha$ ' and ' $\beta$ ' suffixes disappears. We have been unable to detect any difference between phages $\mathrm{d} 8 \beta$ and $\mathrm{d} 10 \beta$ and will call them both $\mathrm{d} 8$. Our version of the structural formulae of types D8, D9, and D10 then becomes $A(d 1)(d 8), A(d 1)(d 9)$, and ?(d1) (d8). The non-lysogenic precursor of type D10, which is indicated by the '?', in the formula, is not known but is probably the same as that of type D7, which appears to be singly lysogenic for phage d1. Type D4 is also singly lysogenic for phage $\mathrm{d} 1$ and we have been unable to identify its nonlysogenic precursor.

The current type strain of D1 is not that originally described by Craigie \& Yen (1938). It is a culture substituted at a later date and originally obtained from Dr P. R. Edwards. This strain will be referred to as D1-E to distinguish it from the original strain which we shall call D1-C. The two strains are not identical in their typing reactions, and those given in Table 1 are those of D1-E. Type D1-C differs from D1-E in being less sensitive to most of the phages and in being completely resistant to phages $D 6$ and $D 11$ at the standard typing temperature. As the temperature is progressively decreased the sensitivity of D1-C to all the $D$ phages is gradually increased, so that at $36.5^{\circ}$ all the reactions except those to phages $D 6$ and $D 11$ are full. These two phages form minute plaques on D1-C at this low temperature but do not, in RTD, cause confluent lysis.

We have found (Bernstein, 1963) that the first $4 \mathrm{hr}$. of incubation of typing plates after the application of phage are the most important in determining the final degree of lysis of D1-C by the typing phages. The reaction on a plate incubated at $38 \cdot 5^{\circ}$ for at least $4 \mathrm{hr}$. will not be affected if it is thereafter transferred to $36 \cdot 5^{\circ}$. Similarly, a reaction established by primary incubation at $36 \cdot 5^{\circ}$ for the same period will not be reduced by later transfer to $38 \cdot 5^{\circ}$. The range of temperature fluctuation during this $4 \mathrm{hr}$. period is critical and should not be more than $\pm 0 \cdot 50^{\circ}$. It has been found that the temperature affects both plaque size and number. Further investigations of this phenomenon are still in progress and a fuller report will be published later.

Strains D1-C and D1-E are both lysogenic and their determining phages are serologically identical. A culture lysogenized with either phage becomes resistant to the other; we shall call this phenomenon reciprocal interference. In spite of the similarities between the two determining phages, it has been found that the differences between the reactions of strains D1-C and D1-E with the Vi-typing phages of the $\mathrm{D}$ group are due to the carriage of phage d1-C by one and d1-E by the other. Lysogenization of type $A$ with phage d1-C $(\rightarrow \mathbf{A}(\mathrm{d} 1-\mathrm{C}))$ produces a culture apparently identical with D1-C; $\mathbf{A}(\mathrm{d} 1-\mathrm{E})$ prepared artificially in the same way is apparently identical with D1-E (Bernstein, 1963). It is not possible to obtain confluent lysis of $\mathrm{A}(\mathrm{d} 1-\mathrm{C})$ at $38.5^{\circ}$ with phages $D 1$ and $D 2$ even by using the two phages in concentrations 10 times higher than their respective RTD.

The other type strains of this group also carry phage d1-C. Most of these strains also display a degree of temperature-dependent variability in phage-typing reactions (Table 3). The carriage of phage d1-C and not d1-E by the doubly lysogenic strains plays a significant role in their type determination. We have found that type 
A made doubly lysogenic with phage d1-E on the one hand and $\mathrm{d} 8$ or $\mathrm{d} 9$ on the other is indistinguishable from type D1-E, whereas the association in type A of d1-C with phages $\mathrm{d} 8$ and $\mathrm{d} 9$ produces cultures identical with $\mathrm{D} 8$ and D9 respectively. It thus appears that phage $\mathrm{d} 1-\mathrm{C}$, but not $\mathrm{d} 1 \mathrm{-E}$, can co-operate with phages $\mathrm{d} 8$ and $\mathrm{d} 9$ in type determination.

Lysogenization of type D7 with phage d8 produces a culture indistinguishable from type D10 and we presume, therefore, that types D7 and D10 have a common non-lysogenic precursor. Lysogenization of type D7 with phage d9 has no detectable effect on the typing reactions at any temperature.

Table 3. The effect of temperature on the typing reactions of members of the $\mathbf{D} 1$ group

\begin{tabular}{|c|c|c|c|c|c|c|c|c|c|c|c|c|}
\hline \multirow[b]{2}{*}{ Vi-type } & \multirow{2}{*}{$\begin{array}{l}\text { Structural } \\
\text { formula }\end{array}$} & \multirow{2}{*}{$\begin{array}{c}\text { Incubation } \\
\text { temperature } \\
\left({ }^{\circ}\right)\end{array}$} & \multicolumn{10}{|c|}{ Typing phages in RTD } \\
\hline & & & D1 & $D श$ & D4 & D5 & $D 6$ & $D^{y}$ & $D 8$ & $D 9$ & $D 10$ & $D 11$ \\
\hline D1-C & $A(d 1-C)$ & $\begin{array}{l}36 \cdot 5 \\
38 \cdot 5\end{array}$ & $\begin{array}{c}\text { CL } \\
+++\end{array}$ & $\begin{array}{c}\text { CL } \\
+++\end{array}$ & $\begin{array}{l}\text { CL } \\
\text { SCL }\end{array}$ & $\begin{array}{c}\text { CL } \\
+++\end{array}$ & ++ & $\begin{array}{l}\text { CL } \\
\text { CL }\end{array}$ & $\begin{array}{l}\text { CL } \\
\text { CL }\end{array}$ & $\begin{array}{l}\text { CL } \\
\text { CL }\end{array}$ & $\begin{array}{l}\text { CL } \\
\text { CL }\end{array}$ & $\begin{array}{c}+++ \\
+\end{array}$ \\
\hline D1-E & $A(d 1-E)$ & $\begin{array}{l}36 \cdot 5 \\
38 \cdot 5\end{array}$ & $\begin{array}{l}\text { CL } \\
\text { CL }\end{array}$ & $\begin{array}{l}\text { CL } \\
\text { CL }\end{array}$ & $\begin{array}{l}\mathrm{CL} \\
\mathrm{CL}\end{array}$ & $\begin{array}{l}\text { CL } \\
\text { CL }\end{array}$ & $\begin{array}{l}\text { CL } \\
\text { CL }\end{array}$ & $\begin{array}{l}\text { CL } \\
\text { CL }\end{array}$ & $\begin{array}{l}\text { CL } \\
\text { CL }\end{array}$ & $\begin{array}{l}\text { CL } \\
\text { CL }\end{array}$ & $\begin{array}{l}\text { CL } \\
\text { CL }\end{array}$ & $\begin{array}{l}\text { CL } \\
\text { CL }\end{array}$ \\
\hline D4 & $?(\mathrm{dl}-\mathrm{C})$ & $\begin{array}{l}36 \cdot 5 \\
38 \cdot 5\end{array}$ & + & - & $\begin{array}{l}\text { CL } \\
\text { CL }\end{array}$ & - & - & - & - & - & - & - \\
\hline $\mathrm{D} 7$ & $?(\mathrm{dl}-\mathrm{C})$ & $\begin{array}{l}36 \cdot 5 \\
38 \cdot 5\end{array}$ & - & + & $\begin{array}{l}\text { SCL } \\
-\end{array}$ & - & - & $\begin{array}{l}\text { CL } \\
\text { CL }\end{array}$ & ++ & - & $\begin{array}{l}\text { CL } \\
\text { CL }\end{array}$ & - \\
\hline D8 & $A(d 1-C)(d 8)$ & $\begin{array}{l}36 \cdot 5 \\
38 \cdot 5\end{array}$ & CL & $\frac{\mathrm{CL}}{-}$ & $\begin{array}{l}\mathrm{CL} \\
-\end{array}$ & $\underline{\mathrm{CL}}$ & - & $\begin{array}{c}\mathrm{CL} \\
-\end{array}$ & $\begin{array}{l}\mathrm{CL} \\
\mathrm{CL}\end{array}$ & ++ & $\begin{array}{l}\text { CL } \\
\text { SCL }\end{array}$ & - \\
\hline D9 & $A(\mathrm{~d} 1-\mathrm{C})(\mathrm{d} 9)$ & $\begin{array}{l}36 \cdot 5 \\
38 \cdot 5\end{array}$ & + & + & $\begin{array}{r}\text { SCL } \\
+++\end{array}$ & ++ & + & $\begin{array}{l}\text { CL } \\
\text { CL }\end{array}$ & $\begin{array}{l}\text { CL } \\
\text { CL }\end{array}$ & $\begin{array}{l}\text { CL } \\
\text { CL }\end{array}$ & $\begin{array}{l}\text { CL } \\
\text { CL }\end{array}$ & ++ \\
\hline D10 & $?(\mathrm{~d} 1-\mathrm{C})(\mathrm{d} 8)$ & $\begin{array}{l}36 \cdot 5 \\
38 \cdot 5\end{array}$ & - & - & ++ & - & - & $\underline{\text { CL }}$ & - & + & $\begin{array}{l}\text { CL } \\
\text { CL }\end{array}$ & - \\
\hline
\end{tabular}

For definition of reactions see Table 1 .

Phages $\mathrm{d} 8$ and $\mathrm{d} 9$ appear to be unrelated to each other and to phages $\mathrm{d} 1 \mathrm{-C}$ and d1-E, and they do not show reciprocal interference. They are both heat labile whereas both varieties of $\mathrm{d} I$ are heat stable. We have not been able to obtain high titre preparations with either phage $\mathrm{d} 8$ or $\mathrm{d} 9$ and plaque counts by either spot or agar-layer titrations are usually less than $10^{5} / \mathrm{ml}$. We have, however, had no difficulty in lysogenizing with these preparations. It has been found (Wilson, unpublished) that there is a disparity between the number of plaque-forming units (PFU) detected by titration and the number of particles, apparently of phage, seen in preparations stained with acridine-orange and viewed by fluorescence microscopy (Anderson, Armstrong \& Niven, 1959). The particle count in terms of PFU is usually one or more log units less than the count under the microscope. This deficiency in plaqueforming ability may be due to a high efficiency of lysogenization with a concomitantly low production of infective phage particles. It has been found that phages $\mathrm{d} 8$ and $\mathrm{d} 9$ do not form detectable plaques at $38^{\circ}$, although lysogenization can be performed at this temperature by blind serial passage. All assays of these phages should be conducted at $36 \cdot 5^{\circ}-37 \cdot 0^{\circ}$.

Read \& Ferguson (1961) state that neither phage d8 nor d9 has any determining ability in type $\mathbf{A}$. This has not been our experience. In fact $\mathbf{A}(\mathrm{d} 8)$ differs from type 
A in that it is completely resistant to typing phage 45 at both 36.5 and $38.5^{\circ}$. At the higher (standard) temperature it also has a much reduced sensitivity to several other typing phages, especially $C 2, C 7, E F, E 9$ and 40 , but these reductions are not evident at $36 \cdot 5^{\circ}$. The distinction between type $A$ and $A(d 9)$ is not so obvious and can only be detected at $38 \cdot 5^{\circ}$. At this temperature $A(d 9)$ is relatively insensitive to typing phage $M 2$. It is interesting to note that we have examined freshly isolated typhoid cultures showing a typing pattern identical with that of $A(d 8)$. These cultures have been found to be lysogenic for a phage indistinguishable from $\mathrm{d} 8$.

Type A has been doubly lysogenized with phages d8 and d9 $(\rightarrow \mathrm{A}(\mathrm{d} 8)(\mathrm{d} 9))$; this culture reacts as if it carried only phage d8. Trebly lysogenized type A (A)(d1-C) (d8) (d9)), however, resembles type D8 at $38.5^{\circ}$ and type D9 at $36.5^{\circ}$. It is evident from this that phages $\mathrm{d} 8$ and $\mathrm{d} 9$ can co-operate independently with $\mathrm{d} 1 \mathrm{-C}$ to produce changes in phage typing pattern; they do not, however, appear to co-operate with each other.

Table 4. Differentiation of the type-determining effects of phages d6-1691, d6-4274, and $f 2$ by the use of three typing phages in RTD

\begin{tabular}{|c|c|c|c|}
\hline \multirow[b]{2}{*}{ Culture } & \multicolumn{3}{|c|}{ Typing phages } \\
\hline & $D 6$ & 29 & 46 \\
\hline $\mathrm{A}(\mathrm{d} 6-1691)$ & CL & SCL & CL \\
\hline$A(d 6-4274)$ & CL & ++ & \pm \\
\hline A(f2) & CL & CL & $\overline{ \pm}$ \\
\hline
\end{tabular}

Thus, phages $\mathrm{d} 8$ and $\mathrm{d} 9$ have type-determining properties, although $\mathrm{d} 9 \mathrm{is}$ very weak in this respect. Cultures lysogenized with them display a degree of temperature-controlled variability of reaction to the Vi-typing phages.

The D6 group. We have included in this group a large number of types: C2, C3, $\mathrm{D} 6, \mathrm{E} 7, \mathrm{E} 9, \mathrm{~F} 2, \mathrm{~K} 2, \mathrm{M} 3$ and 29. The criterion for inclusion has been that the strains should be lysogenic for either phage $\mathrm{d} 6$ or phage f2. These two type-determining phages were first described by Felix \& Anderson (1951) and Anderson \& Felix $(1953 b)$ who found that they were serologically identical and that they resembled each other in host-range, plaque morphology and heat sensitivity; they also displayed reciprocal interference. Differentiation between the two phages could be made only on the basis of their type-determining activity. It was found that $A(d 6)$ was sensitive to phage $D 6$, whereas A(f2) was fully sensitive to phage 29 in addition to phage $D 6$ (see Table 4 ).

In the survey of various types made by Anderson \& Fraser (1955) it was shown that the structural formulae of types $\mathrm{C} 2$ and $\mathrm{C} 3$ were $\mathrm{Cl}(\mathrm{d} 6)$ and $\mathrm{Cl}(\mathrm{f} 2)$ respectively. The difference in phage sensitivity between these two types is analogous to that between types $\mathrm{D} 6$ and 29 , i.e. $\mathrm{C} 2$ is sensitive only to phage $\mathrm{C} 2$, whereas $\mathrm{C} 3$ is sensitive to phages $C 2$ and $C 3$. Anderson \& Fraser also found that the structures of types E7 and E9 were E1(f2) and E1(d6) respectively.

There are, therefore, three pairs of types which have resulted from the lysogenization of precursor types $\mathrm{A}, \mathrm{Cl}$, and El with either phage d6 or f2. However, type F2, which has the structural formulae F1(f2), has not so far been paralleled by a naturally occurring type with the formula F1(d6). Such a culture can nevertheless 
be produced in the laboratory. Similarly, types D11, K2 and M3, all lysogenic for a phage resembling d6, have so far no counterparts lysogenic for f2.

It was reported by Anderson \& Felix (1953b) that the phages, called by them $30^{\prime}$ f2, and $29^{\prime}$ isolated from types C3 (at that time designated type 30), F2, and 29 were identical in type-determining efficiency but differed in the resistance which they produced to phages other than the adapted typing preparations. For the purposes of this paper we will refer only to phage f2. It has been found (E. S. Anderson, unpublished) that there are also differences between some of the phages $\mathrm{d} 6$ isolated from different lysogenic types. There are, for example, two members of this phage 'family' which have been isolated from the different type strains of $\mathbf{D} 6$. One which we will call $\mathrm{d} 6-1691$ is found in culture $\mathrm{T} 1691$, which was the original type strain of type D6. The other phage d6-4274 was isolated from a culture, $\mathrm{T} 4274$, which was substituted for the type strain at a later date. These two cultures differ in that D6-1691 is sensitive in moderate degree to a wide range of phages which do not, at RTD, lyse D6-4274. These differences between the two strains have been found to be due to the different $\mathrm{d} 6$ determining phages which they carry. In many respects phage $\mathrm{d} 6-1691$ appears to possess a determining ability about halfway between those of $\mathrm{d} 6-4274$ and $\mathrm{f} 2$. Thus $\mathrm{A}(\mathrm{d} 6-1691)$ and $\mathrm{D} 6-1691$ are partially sensitive to phage 29. A full differentiation between the determining powers of these phages can be made by lysogenizing type $A$ with each and testing the resulting cultures with typing phages $D 6,29$, and 46 (Table 4 ). It is probable that there are more forms of $\mathrm{d} 6$ than the two we have examined. Indeed, it is probable that there is a group of related phages possessing a spectrum of similar determining abilities ranging from that of $\mathrm{d} 6-4274$ to that of $\mathrm{f} 2$.

We have found that all the type strains so far examined of the d6-determined types, other than D6-4274, carry the 1691 variant, or ones closely resembling it. Representatives of these types carrying $\mathrm{d} 6-4274$ may occur in nature but none has been encountered by us hitherto. In the cases of types $\mathrm{C} 2$ and $\mathrm{E} 9$, such alternative types have been made by lysogenizing types $\mathrm{C} 1$ and $\mathrm{E} 1$ respectively with phage d6-4274. These 'artificial' types differ from the natural type strains in their higher degree of specificity.

The non-lysogenic precursors of types D11 and K2 have not yet been isolated. It is possible, however, to produce a culture resembling type $\mathbf{K} 2$ by lysogenization of type $\mathrm{K} 1$ with phage d6. Culture $\mathrm{K} 1$ (d6) differs from $\mathrm{K} 2$ in that it is lysogenic for phage $\mathrm{k}$ as well as $\mathrm{d6}$. Since phage $\mathrm{k}$ is not known to possess any determining power it is possible that a non-lysogenic type $\mathrm{Kl}$ could exist and that this is the precursor of type K2. It has not yet been possible to synthesize a culture resembling type D11.

All the typing phages of this group are either pure host-range mutants (phages $D 6, D 11, K 2$, and 29 ) or phenotypically modified mutants (phages $C 2, C 3, E 7, E 9$, F2 and M3). The phenotypic modifications represented are identical with those produced by types $\mathrm{C} 1$ (in phages $C 2$ and $C 3$ ), $\mathrm{E} 1$ (in phages $E 7$ and $E 9$ ), $\mathrm{F} 1$ (in phage $F 2$ ) and $M 1$ (in phage $M 3$ ). The mutations occurring in phages $C 2, D 6, E 9$ and $M 3$ appear to be identical and we shall call them the $D 6$ mutation. Similarly, those found in phages $\mathrm{C} 3, \mathrm{E}^{7}, \mathrm{~F} 2$ and 29 may be designated the 29 mutation. The mutations represented by phages $D 11$ and $K 2$ are distinct from each other and from the $D 6$ and 29 mutations. It is possible to distinguish between the basic mutations by an examination of their host ranges on a small number of types (Table 5). 
T Group: types E10, F5, T, 28 and 39. Felix \& Anderson (1951) showed that the specificity of type $T$ was due to a determining phage, $t$, and its structural formula was represented by Anderson \& Fraser (1955) as A(t). Phage $t$ was found to be very unstable even at $4^{\circ}$, and we do not now have an authentic strain of the original phage $t$ in our possession.

Phage $T$ was found by Anderson \& Felix (1953b) to represent a phenotypically modified adaptation of Vi-phage II. Work by Anderson \& Fraser (1955) suggested that the Vi-typing phages specific for lysogenically determined cultures were host range mutants of Vi-phage II. There was thus a discrepancy in the case of phage $T$ which seemed from earlier experiments to be modified in phenotype only.

Table 5. Differentiation of the D6, D11, K2 and 29 mutations of Vi-phage II by an examination of their lytic activity, in RTD, on a restricted number of indicator strains

Basic mutants of Vi-phage II*

\begin{tabular}{|c|c|c|c|c|}
\hline Vi-types & $D 6$ & $D 11$ & $K 2$ & 29 \\
\hline D6-4274 & CL & CL & CL & ++ \\
\hline D11 & - & CL & - & - \\
\hline 29 & CL & CL & CL & CL \\
\hline 42 & - & CL & CL & +++ \\
\hline
\end{tabular}

It was later shown by E. S. Anderson \& A. Fraser (unpublished) that the situation is much more complex. They found that single plaque selection of titration of phage $T$ on type A gave lines of phage with a wide range of EOP on type T, that is, the ratios of plaque titres on type $\mathbf{T}$ to those on type $\mathrm{A}$ were highly variable. The EOP of some lines were close to that of phage $A$, that is, $c .10^{-5}$; others resembled phage $T$, and had an EOP of $0 \cdot 5$; and yet others occupied intermediate positions, the range from one extreme to the other being apparently continuous. It seemed as if phage $T$ represented a change in host-range of Vi-phage II intermediate in position between host-induced modification and mutation.

We have re-investigated type $T$ and an unexpected finding has been that we have been unable to isolate any temperate phage from the type strain or from several other cultures belonging to the type. We have reason to believe that the strain is still lysogenic, but that the prophage has become defective, that is, it no longer produces mature plaque-forming phage particles. An indication that this is so is provided by the analysis of types E10 and F5.

Type F5 is lysogenic and the temperate phage, f5, appears to possess all the properties originally ascribed to phage $t$. It displays the same degree of instability, has the same plaque morphology, and is fully neutralized by an antiserum prepared by Anderson \& Felix (1953 $b$ ) against phage t. Type A lysogenized with f5 is indistinguishable from type $\mathrm{T}$ and type $\mathrm{T}$ is insensitive to phage $\mathrm{f5}$. On these grounds we suggest that phages f5 and $t$ are the same; we shall designate both phage $t$.

Type F1 lysogenized with $t$ is identical with F5. The structural formula of F5 can be represented, therefore, as F1(t). Type E10 has not been found to be lysogenic, but it is resistant to phage $t$. Lysogenization of type $\mathbf{E} 1$ with phage $t$ produces a 
culture which reacts as type E10. This suggests that the structural formula of E10 is probably $\mathbf{E} \mathbf{1}(\mathrm{t})$ but that, as in type $\mathbf{T}$, the determining phage is defective.

Typing phage $F 5$ appears to be a pure host-induced modification of Vi-phage II and attempts to adapt phage $T$ to F1 have hitherto yielded only phage $F 1$. Phage $E 10$, however, has been found to display a similar diversity of adaptation to that described earlier in phage $T$ (B. Parkhouse, unpublished). Any population of phage E10 yielded some lines behaving as phenotypically modified mutants; such lines produce a phage similar to $T$ after de-adaptation, and revert to phage E10 when re-adapted on type E1. Another portion of the same population was a pure hostinduced modification, so that phage $E 10 / A$ was phage $A$ and phage $E 10 / A / E 1$ was phage $E 1$. Attempts to adapt phage $T$ to type $\mathbf{E} 1$ have so far yielded only phage $E 1$.

The mutation represented in phage $E 10$ is, however, not the same as that in phage $T$ in that phage $E 10$ can lyse type 28, which phage $T$ cannot; moreover, the hostrange of the mutant form of phage E10/A is indistinguishable from that of phage 28 (see below). It is apparent that there is a diversity of changes possible in the adaptation of Vi-phage II to complexes carrying prophage $t$.

There are two other types that must be considered as part of this group, in that, in both cases, the typing phage was derived from phage $T$. The first of these types is 28 , which was shown to be lysogenic by Anderson $\&$ Felix (1953b). These authors were not able to show that temperate phage $28^{\prime}$ possessed determining powers. We shall consider this aspect of the type in the next section (see types B3, C9, and 28 ). Typing phage 28 has an equal EOP on types $\mathbf{T}$ and 28 , that is, about $10^{-1}$ of that on type A. This EOP is unchanged by growth in type A. The phage is, therefore, a mutant arising in a stock of phage $T$.

The remaining type, 39 , has not been shown to be lysogenic. The typing phage has an EOP on type 39 equal to that on type $T$ and about 0.5 of that on type $A$. On type 28 the EOP is $10^{-2}$ of that on $\mathrm{T}$ and 39 . After de-adaptation the resulting phage has an EOP on type 39 only $10^{-3}$ of that on $\mathbf{A}$ or $\mathrm{T}$. It thus appears that the phage is the result of a host-induced modification of phage $T$. The type strain is resistant to phage $t$ and it is possible that it is lysogenic for defective phage $t$. No precursor type is known which produces type 39 after lysogenization with phage $t$.

Types B3, C9 and 28. These three types are lysogenic; their temperate phages display reciprocal interference and are serologically identical; the carried phages are designated b3, c9, and $28^{\prime}$ respectively. It was not possible in earlier work to show that phages b3 and $28^{\prime}$ are type-determining (Anderson \& Felix, 1953b; Anderson \& Fraser, 1955; Ferguson, Juenker \& Ferguson, 1955) but we now have evidence that they possess this property. All three phages of this group are similar in their determining powers. They will convert type $\mathrm{A}$ into type $\mathrm{B} 3$ and type $\mathrm{C} 1$ into $\mathrm{C}$. In addition, they will convert type $\mathrm{T}$, or $\mathrm{A}(\mathrm{t})$, into type 28 . We use separate symbols for them, however, because there are small differences in their type-determining properties. It is possible that they represent only different varieties of the same determining phage, which could then be called b3. The structures of types B3, C9 and 28 are at present represented as $\mathbf{A}(\mathrm{b3}), \mathrm{C1}(\mathrm{c} 9)$ and $\mathbf{A}(\mathrm{t})$ (28) respectively.

Typing phage $B 3$ is a pure host-range mutant whereas phage $C 9$ is a phenotypic modification of a mutant, de-adaptation of which yields a phage identical with phage $B 3$. Adaptation of phage $C 9 / A$, or of phage $B 3$, to type $\mathrm{C} 1$ yields phage $C 9$. Phage $C 9$ may therefore be assumed to be a $C 1$ modification of phage $B 3$. 
Phage 28 has been considered earlier and no relationship can be found between it and phages $B 3$ and $C 9$.

Type J2. This type is lysogenic and the temperate phage which it carries, j2, is serologically distinct from all others in our possession. Phage $\mathrm{j} 2$ resembles $\mathrm{d} 1 \mathrm{in}$ plaque morphology and heat stability, but does not show reciprocal interference with it or with any of the other temperate phages we have examined.

Lysogenization of type $\mathrm{J} 1$ with phage $\mathrm{j} 2$ produces a culture indistinguishable from type J2 and, although we have not been able to isolate a non-lysogenic precursor from the type strain, we consider its structural formula to be $J 1(\mathrm{j} 2)$. When type $\mathbf{A}$ is lysogenized with phage $\mathrm{j} 2$ the resultant culture resembles type D5, but differs from it in that $A(j 2)$ is sensitive to typing phage $J 2$ whereas type D5 is not, and that D5 is sensitive to $\mathrm{j} 2$ whereas $\mathrm{A}(\mathrm{j} 2)$ is obviously not.

Table 6. Efficiencies of plating $\left(E O P^{*}\right)$ of three different mutant phages produced by the adaptation of phage A to Vi-type $\mathrm{J} 2$

\begin{tabular}{lccc} 
& \multicolumn{3}{c}{ Phage $A / J 2$} \\
Vi-type & $\overbrace{\text { Mutant 1 }}$ & Mutant 2 & Mutant 3 \\
D1-E & $1 \cdot 0$ & $0 \cdot 1$ & $0 \cdot 3$ \\
D5 & $1 \cdot 0$ & $<10^{-6}$ & $0 \cdot 2$ \\
J2 & $1 \cdot 0$ & $1 \cdot 0$ & $1 \cdot 0$ \\
42 & $<10^{-6}$ & 0.3 & $0 \cdot 3$
\end{tabular}

* EOP $=$ the ratio of the plaque titre obtained on a given type to that on type $A$.

We have not been able to demonstrate lysogenicity in the D5 type strain. Ferguson et al. (1955) reported the isolation of a phage, which they designated d5, from a culture which appeared to belong to type D5. Phage d5 was serologically identical with phage $\mathrm{d} 1 \mathrm{but}$ did not reciprocally interfere with it. They also found that the D5 type strain was sensitive to d5. When they lysogenized type A with d5 the resulting culture behaved as type D1. It is possible that their identification of A(d5) as type D1 was an error. Anderson \& Fraser (1956) showed that the selection of plaques from titration of phage $A$ on type D1 yielded approximately equal proportions of mutant lines corresponding to phages $D 1$ and $D 5$. It was found that the 'phage $D 1$ ' in current use at that time was in fact a $D 5$ mutant which obviously could not distinguish between types $D 1$ and $D 5$.

We do not know the true relationship between type D5 and cultures $A(d 5)$ and $A(j 2)$. It is interesting, however, that both D5 and $\mathbf{A}(\mathrm{j} 2)$ become resistant to typing phage $D 9$ if examined at $36 \cdot 5^{\circ}$. We do not have a culture of $A(d 5)$ and so do not know whether it displays a similar variability.

Typing phage $J 2$ is a phenotypic modification of a hitherto undescribed mutant of Vi-phage II. After de-adaptation the phage will lyse type A and also A(j2), and when re-adapted to type $J 1(=J 2 / A / J 1)$ it regains the full lytic activity of phage $J 2$. It has been found that adaptation of Vi-phage II to type J2 yields, in equal proportions, three different phages, all of which are phenotypically modified mutations. The host-induced modifications in each seem to be the same as that produced by type $\mathbf{J} 1$, but the mutational components differ. Table 6 shows the EOP of the three mutants on types A, D1, D5, J2 and 42. All the phages lyse types A, J1, and J2. In addition, one phage lyses types D1 and D5 fully whereas a second lyses types 
D1 and 42 but with a reduced degree of reaction. The third mutant lyses types D1, D5 and 42 with equal but reduced efficiency. The second mutation is that selected for in the preparation of the current typing phage.

Although the three mutants that lyse J2 also lyse D1, none is identical with the $D 1$ mutation. Adaptation of phage $D 1$ to type $\mathrm{J} 1$ does not yield a phage with the ability to lyse J2. However, phage $D 5$ adapted to $\mathrm{J} 1$ produces a phage closely resembling mutant 1 of Table 6 .

Types E2 and L2. Anderson \& Fraser (1955) stated that these phages were pure phenotypic modifications of Vi-phage II reverting to phage $\boldsymbol{A}$ on de-adaptation. Further work has shown, however, that the phages concerned are phenotypically modified host-range mutants. If phage E2/A is propagated in type $\mathrm{E} 1(\rightarrow \mathrm{E} 2 / \mathrm{A} / \mathrm{E} 1)$ the resultant phage is $E 2$ and not $E 1$, as would be the case if $E 2 / A$ was phage $A$. Similarly, phage L2/A grown on type L1 becomes phage $L 2$.

We have found that the mutation represented in phage $E 2$ is the same as that in phage $C \%$. There is no phage in the Vi-typing scheme which represents the pure mutant form of phages $E 2$ and $L 2$, nor are there accepted types other than $A$, which are sensitive to phage $E 2 / \mathrm{A}$ or $L 2 / \mathrm{A}$.

As was reported by Anderson \& Fraser (1955) these types appear to be nonlysogenic.

Types E3 and E4. The typing phages for both these types are host-modified mutants. Neither culture appears to be lysogenic. In each case the host-induced modification is the same as that represented in phage $E 1$.

The mutational component of these phages has been previously investigated by Scholtens (1956) who suggested that they might both be phenotypically modified D1 mutations. We cannot fully characterize the mutations represented in these typing phages but we do not believe them to be $D 1$, or at least not the same mutation as that found in the $D 1$ typing phage. They are, however, clearly related to this mutant.

Both types are sensitive to phages d1-C and d1-E, which indicates that they are not lysogenic for either phage even in defective form. Lysogenization of type E4 with either form of $\mathrm{d} 1$ produces no demonstrable effect on typing pattern. When type E3 is lysogenized with phage d1-C, however, it loses its sensitivity to typing phage E3 (E.S. Anderson, unpublished); phage d1-E however, has no obvious effect in type E3. The change following lysogenization with d1-C is consistent with a prophage co-operation effect such as had been found in several members of the D1 group. No other evidence has been found for a temperate phage in type E3.

Phage $E 3$ lyses type D1 in addition to its type strain and it might appear from this, and from the fact that the de-adapted phage still lyses D1, that the basic mutation in this phage is that represented in phage D1. However, phage D1 adapted to type E1 produces a phage different from phages $E 3$ or $E 3 / A / E 1$. Phage D1/E1 lyses E3(d1-C) in addition to A, D1 and E3, whereas, as stated above, phage E3 will not attack strain E3(d1-C). Phages E3 and D1/E1 also lyse E1(d1-C) (E. S. Anderson, unpublished) and E1(d1-E). These two lysogenized cultures resemble type E3 in their sensitivity patterns.

Phage $E 4$ lyses types D1 and E3 in addition to types A and E4. It has not been possible to obtain from phage $D 1$ a host-induced modification which has the ability to lyse type E4. Nor has it been possible by lysogenization to produce from type E1 a culture resembling E4. 
It is evident that the phenotypic change in phages $E 3$ and $E 4$ is the same as that in phage $E 1$. The mutational change is different in each phage and neither is the same as that of phage $D 1$, although de-adapted phages $E 3$ and $E 4$ will lyse type D1. There are, however, several known mutants other than $D 1$ which have the latter property (e.g. D5, D6). The specificity of the type strains E3 and E4 is certainly not due to the carriage of either of the forms of phage $\mathrm{dl}$ described earlier.

\section{DISCUSSION}

Our investigation of the various Vi-types and typing phages, both those previously examined and those more recently described, has produced results essentially in agreement with earlier findings and with the hypotheses of type determination and Vi-phage II adaptation as summarized by Anderson (1962a). Any additional information has added to, rather than contradicted, the findings of the earlier investigations of Anderson and his co-workers.

Several of the newer types are lysogenic for phages previously described. The results obtained with these types have been fully in accordance with predictions that could have been made from the arguments of Anderson \& Fraser (1955) which were based on fewer types.

It may be that several types which we have stated to be non-lysogenic do carry phages, but that our methods are not adequate for their detection. We have suggested that some types, for example E10, are lysogenic for a defective prophage since we have been able to synthesize such types by the lysogenization of suitable precursors with active phage preparations. Defectivity may be the cause of apparent non-lysogenicity in other types, but we have no means of verifying this.

Anderson (1959) has shown that when type $A$ is lysogenized with phage $25^{\prime} \mathrm{c}$, a clear-plaque variant of $\mathbf{2 5}^{\prime}$, clones may be isolated which do not yield any temperate phage although they react as type 25 with the Vi-phages and are resistant to phage 25'. It thus seems that the mutant of $25^{\prime}$ they carry has become defective. It is also known from the earlier published work of Anderson \& Felix (1953b) that type $T$ is lysogenic. However, we have recently been unable to isolate phage $t$ from the type strain of $\mathbf{T}$ or from several other representatives of it, although we have isolated a phage which appears to be identical with $t$ from type F5. These results suggest that phage $t$ has become defective in the type $T$ strains examined.

Even when a phage is not defective it may be difficult to detect. Several of the temperate phages form extremely minute, shallow, opaque plaques. Their plaqueforming ability may also be very sensitive to environmental conditions. The plaque-forming efficiency of a temperate phage must be inversely related to its efficiency of lysogenization. A phage with an efficiency of lysogenization approaching $100 \%$ would obviously form plaques very rarely. We have already mentioned the evidence that the plaque counts of phage $\mathrm{d} 8$ on type $\mathbf{A}$ at $36 \cdot 5^{\circ}$ may be only onetenth, or less, of the true phage particle content of $\mathrm{d} 8$ lysates. Several of the known determining phages give rise to mutants with a variety of lysogenic abilities (Anderson, 1959). Some of these, such as $25^{\prime}$ c, form clear plaques but are not truly virulent because they lysogenize with low efficiency. Other mutants have a high rate of lysogenization and form very turbid plaques.

It is known from studies of other phages ( $\operatorname{such}$ as $\lambda, \mathrm{P1}$ ) that cultural modifications will alter the efficiency of lysogenization (Lieb, 1953; Bertani \& Nice, 1954; Fry, 
1959). Similar studies have not been made with the determining phages carried by Salmonella typhi but they may exhibit the same phenomenon. We have routinely used two different incubation temperatures and a variety of indicator strains, but only one medium, in our endeavours to isolate temperate phages. However, these conditions may not be adequate for the detection of all temperate phages.

The phages isolated may be broadly classified into those with type-determining ability and those without it, phage $\mathrm{k}$ being an example of the latter category. The statement that a given phage has no type-determining power is only true within the framework of reference provided by the Vi-phage typing scheme in its present state. It is possible that the discovery of new typing phages may detect a type-determining property in phages now thought to lack this.

Those phages possessing a determining power may be further subclassified by a consideration of their type-converting efficiency. Many of the phages, such as $\mathbf{2 5}^{\prime}$, may be called fully determining, since lysogenization of type $\mathrm{A}$ with one of them produces a highly specific type. Others, which we shall call semi-determining (Anderson \& Williams, 1956), do not produce clear-cut types when lysogenizing type $\mathrm{A}$. The alteration in the phage sensitivity of type A may consist solely in the reduction, or loss, of one or more reactions. If a semi-determining phage is used to lysogenize a culture already possessing a restricted phage sensitivity spectrum, it may have a more specific effect.

Some of these semi-determining phages produce an effect only in cultures already lysogenic for another, unrelated phage. Phage ds produces a minimal, although characteristic change in the typing pattern of type A. If, however, d8 is used to lysogenize type D1-C, or A(d1-C), the resultant culture resembles type D8. The result of this double lysogenization is apparently not a simple summation of the Vi-phage blocking power of the two determining phages. It must be the result of some form of co-operation or synergism between them. This co-operation appears to be highly specific, since phage d8 will interact with phage d1-C but not with the closely related d1-E in the lysogenic cells.

One must distinguish between those synergistic effects shown by phages $\mathrm{d} 8$ and d1-C and the additive effects which may be obtained with other double lysogenizations. If type D1-E or A(d1-E), is lysogenized with phage d6, the resultant culture resembles type D6 (Jude, Nicolle \& Ducrest, 1951). It is not, however, identical with $\mathrm{D} 6$ in that it is resistant to typing phages 36 and 40 . The Vi-phage resistances of $A(\mathrm{~d} 1-\mathrm{E})(\mathrm{d} 6)$ may be predicted by a superimposition of the typing patterns of types D1 and D6, remembering that resistance is dominant. In some cases, double lysogenization will yield a culture completely insensitive to all the available typing phages. A summation effect is detectable only when the determining abilities of the two phages are suitably complementary.

Some phages with full determining powers may lack a detectable determining effect in certain cultures. For example, types 36 and 40 may be lysogenized with phage $\mathrm{d} 6$ without any apparent effect on their typing reactions. The reasons for this are obvious from a study of the phage sensitivities of types 36, 40 and D6 in Table 1. It can be seen that types 36 and 40 are already resistant to typing phage $D 6$ and its modifications, although phages 36 and 40 will both lyse type $\mathrm{D} 6$, the reaction pattern of which is determined by $\mathrm{d} 6$. Some cultures of type 40 are lysogenic for phage d6. 
The type-determining phages appear to have no effect on their hosts other than to modify their sensitivity to the typing adaptations of Vi-phage II. Any other characteristic of a given precursor, such as its biotype, is found in all its lysogenic descendants. For instance, all the members of types M2 and M3 which have been examined are non-fermenters of xylose, as is the precursor type M1 (Nicolle, Hamon \& Diverneau, 1962). Characters unchanged by lysogenization, such as drug resistance, may be used to obtain type conversion with determining-phages which are difficult to isolate. Mixed cultures, in broth, are made of a streptomycinresistant strain of type $A$ and of a streptomycin-sensitive culture of the type thought to carry the determining phage. After a period of growth the mixed culture is plated on streptomycin agar and the resistant colonies tested for any change in phage type.

All lines of Vi-phage II, whether resulting from mutation or from host-induced modification, are absorbed by, and will kill, any Vi-positive strain of Salmonella typhi (Anderson \& Fraser, 1956; Anderson, $1962 a$ ), although there may be no multiplication of the phage. The fact that Vi-phage II is active in transduction (Baron, Formal \& Spilman, 1953) suggests that the phage DNA enters the bacterium even in cells which do not support phage growth.

Since all adaptations of Vi-phage II adsorb to any Vi-positive strains of Salmonella typhi to produce a lethal effect, one of the most valuable techniques for the detection of phage recombination, the use of mixed indicators, cannot be used in the genetic analysis of Vi-phage II. This is because all lines of phage, parent or recombinant, mixed or pure, produce the appearance of clear plaques on mixed indicator cultures. For these reasons the loci concerned in the adaptation of Vi-phage II to the many types of $S$. typhi have not yet been mapped.

In several other phage-host cell systems it has been shown that cultures able to induce phenotypic modification in the phage are lysogenic. It has been suggested that the modification is the result of interaction between the infecting phage and the temperate prophage (Lederberg, 1957; Christensen, 1961 ; Drexler \& Christensen, 1961). There is no present evidence that the host-range modifications of the Vityping phages, whether of mutational or host-induced origin, are due to genetic interaction between Vi-phage II and type-determining phages.

Vi-types which fall into groups in the typing scheme (see Table 1) are so classified because they react as though they possessed a common precursor. Thus, types $\mathrm{C} 8$, E8 and 26, although they each carry phage $26^{\prime}$, fall into three different groups in the typing scheme because they have different precursors $(\mathrm{C} 1, \mathrm{E} 1$ and $\mathrm{A}$, respectively). They could, however, be grouped together because they carry phage $\mathbf{2 6}^{\prime}$.

If we were to group types according to the carriage of the same determining phages, the specific typing phages of each group would usually prove to be different phenotypic modifications of the same host-range mutant, the host-induced modifications being those corresponding to the precursor types concerned. There are, however, some groups which are determined by a single temperate phage, but in which the typing phages for the individual types are mostly distinct host-range mutants. These mutants are not identical but are evidently related to each other. Such an example is found in some members of the $\mathbf{D}$ group of Vi-types.

As is evident from an examination of the typing scheme (Table 1), many of the types are sensitive to a number of mutants of Vi-phage II. Such types, therefore, 
select these mutants indifferently from wild stocks of Vi-phage II, that is from phage $A$, and 'adaptation' of phage $\boldsymbol{A}$ to these types without the subsequent characterization and purification of phage clones may result in the production of a mixture of phages of different specificities. As has been mentioned earlier, typing phage $J 2$ can be represented by any one of at least three different variants of Vi-phage II and the choice of one of these as the typing phage is relatively arbitrary. From the point of view of practical phage-typing convenience it is usual to choose that phage with the most restricted host-range, that is, lysing as few types as possible other than the homologous one.

This possible heterogeneity in the typing preparations is of practical importance in the development and application of the typing scheme. Care must be taken in the maintenance and propagation of phage stocks to ensure completely homogeneous continuity. In the development of a new typing phage the possibility of heterogeneity in the final preparation must be fully investigated. Such heterogeneity may be minimized by testing single-plaque lines in order to select those with the most restricted host-range.

Adaptation may also be carried out by the prolonged joint growth in broth of phage $\boldsymbol{A}$ and the cultures under investigation. This may be continued for long periods. Under these conditions heterogeneity in the resultant phage is very likely to occur, especially if the culture is sensitive only to mutants of the parent phage. The relative proportions of the various mutants in the final lysate will bear a direct relationship to their frequency in the starting phage and to their rate of growth and burst-size in the type concerned. Additional mutations may also occur during successive cycles of growth and may be represented in the final lysate.

The host range of typing phages is usually ascertained at a selected RTD. This dilution is used because it yields a reaction approaching the maximum on homologous types whilst minimizing cross-reactions. For purposes other than those of routine phage typing, however, it may be better to characterize the phages not in terms of their activity at the RTD but by a consideration of their EOP on the various indicator strains.

Several of the typing phages are mutants which produce small or minute plaques on their homologous host strains but not always on others. In order to obtain confluent lysis with such a phage it may be necessary to use a high concentration of phage particles, whereas with a large-plaque-forming phage a lesser number would be required. Thus, the RTD of phage 38 contains $c .10^{6}$ phage particles $/ \mathrm{ml}$. whereas that of phage 41 , which has a larger plaque size, contains only $10^{3}$. The fewer particles a phage contains at RTD the fewer cross-reactions it will give. In addition, a phage which requires $10^{6}$ particles $/ \mathrm{ml}$. to produce confluent lysis of its host may cause difficulty in type diagnosis due to the non-specific lethal effect of such a high phage concentration on the bacteria to which it is applied, even if lysis does not occur. This effect is enhanced when several different phages are pooled in order to reduce the number of reagents in routine use. For this reason, care must be taken in the construction of such pools to ensure that their phage content in terms of total particles/ml. is not excessive. Not only may the RTD of different typing phages contain vastly different amounts of phage, but the RTD of any given phage may also vary according to the circumstances under which it is estimated. There are several factors which may affect the sensitivity of the homologous type strain, as 
well as of others, towards a given typing phage. Some of these may be intrinsic to the cultures whereas others may be environmental in nature-type of medium, temperature of incubation and so on.

It is often found that the sensitivity of a given type may fluctuate over a period of time. Many types on storage gradually become more resistant to their specific typing phages. The character of the lysis may also change. For example, it may become more opaque because an increase of resistance of the host strain because of an accumulation of Vi-negative or other variant bacterial forms. In such cases it is often possible to regain a fully sensitive (Vi-positive) culture by single colony selection.

Those types which are lysogenically determined may, on storage, lose their determining phages with a consequent change in typing pattern. This is more common with certain phages such as those of the d6-f2 group (Anderson, 1951). Strains which are losing their determining phage become progressively more sensitive to heterologous typing phages. When this occurs it may be possible to re-isolate the specific type strain by single-colony selection. If this fails it may be possible to regain the type by relysogenizing the culture with the requisite phage. Types which are apparently non-lysogenic may display a loss of specificity for reasons at present unknown. This loss may be stepwise in nature, in which case the change in sensitivity to the typing phages resembles that found when a determining phage is lost in a lysogenically determined type. Or it may be gradual, with the widespread acquisition of sensitivity to heterologous typing phages; this change is known as 'degradation'.

In addition to those fluctuations in sensitivity to the Vi-typing phages which may be directly attributed to the culture of a type strain, there may be others which are due to the environmental conditions of the test. In general, anything which causes a decrease in the growth rate of the culture, such as unsuitability of the nutrient medium, will lead to a reduction in phage sensitivity. Conversely, an improvement in growth conditions may result in better lysis.

It is important, therefore, to standardize fully the cultural conditions for selection of the RTD of a phage and for routine phage-typing. For these purposes the medium chosen should display minimal batch-to-batch variation but need not be optimal for growth. The medium which we have used in this survey, and which is that recommended for routine Vi-phage typing, that is Bacto nutrient broth, is certainly not the most nutritious for Salmonella typhi. However, it has been remarkably constant during the course of the 20 years during which batch-to-batch comparisons have been made. If phage-typing has to be conducted on a different medium, it is necessary to determine the phage RTD's on it.

If the medium to be used is one which is liable to variation in composition it is advisable to set aside a large quantity derived from either a single production run or from the pooling of several runs. Individual batches should be 'standardized' by comparison with a reference medium.

The reactions listed in Table 1 represent the 'mean' of a number of typing tests. Because of the virtual impossibility of exactly duplicating environmental conditions, the precise reproduction of the reactions listed is almost impossible, however closely the prescribed technique is adhered to. Some of the discrepancies may be due to variation in plaque size, so that reactions have become more or less obvious. Many of the cross-reactions listed are at the limit of visibility with a $10 \times$ 
hand lens and it follows that alteration in plaque size will grossly affect these. It is obvious from the results presented on type $\mathrm{D} 8$ that phage typing must be conducted at a well stabilized temperature of $38 \cdot 5^{\circ}$.

During the phage typing of large numbers of cultures, some are found which differ in only a small degree from some of the listed types. Cultures resembling A but resistant to phage $C 5$ or 45 are known, as are cultures like $\mathrm{C} 1$ but resistant to phage C5. Some of these cultures are known to be lysogenic for semi-determining phages such as d8. It is difficult to decide on suitable designations for such cultures, whose typing patterns are as distinct and stable as those of the established types. They would formerly have been classified as 'degraded' but we do not think that this is justifiable. It might be preferable to regard them as subtypes, or varieties, of the types which they most closely resemble.

It is common practice in most typing laboratories to pool groups of phages for routine phage-typing in order to limit the number of phage preparations that have to be applied to a culture. The use of such pools economizes in time and media but may unfortunately lead to errors in type diagnosis by concealing the slight differences in reaction to which we have referred. In particular, a culture may be classified as type $\mathbf{A}$ when in fact the use of phage pools has concealed the loss of one reaction, say, to phage $C 5$. The true reaction of such a culture can only be detected by the use of all the typing phages individually. It is now our routine practice so to examine any culture which appears to be type $\mathbf{A}$.

There are other cultures which are sensitive in varying degree to many of the typing phages but which show no obvious relationship to any of the standard types. These strains may nevertheless possess a typing pattern which is sufficiently distinct and stable to be valuable for epidemiological purposes. Some of these strains may be descended from 'type $\mathbf{A}$ which has been lysogenized with one of the semi-determining phages mentioned earlier. A few of the existing members of the typing scheme, such as B1 and 45, have typing patterns which might justify their being called degraded. The typing phages corresponding to these strains are, however, highly specific, and it may be possible to obtain equally specific phages for other strains diagnosed as degraded.

It is possible to synthesize many new types by the lysogenization of various precursors with any of the determining phages to which they are sensitive. Many such laboratory 'types' have already been made, and some of them have later been isolated in nature.

We have thought it useful to summarize the information at present available concerning type-determination in Salmonella typhi and the host-range specificities of the typing adaptations of the Vi-phage II. This information provides a basis for the better understanding of the principles of Vi-phage typing of $S$. typhi. It is unfortunate that there has been no recombinational analysis of the many host-range adaptations of Vi-phage II. Because of the technical difficulties mentioned earlier, we do not know whether the host-range mutations are located at one or many chromosomal sites, and, if the latter, whether more than one cistron is involved. We are hopeful, however, that a suitable technique will be evolved which will make such analysis possible. Vi-phage II might then be a useful organism for the analysis of genetic fine structure. 
We are grateful to Dr E. S. Anderson for his advice and helpful criticism during the course of these investigations and of the preparation of this paper. We also wish to thank him and Mrs B. Parkhouse for permission to quote from their unpublished results. The technical assistance of Mrs K. Kiewe and Mr F. Flynn is gratefully acknowledged.

\section{REFERENCES}

Adams, M. H. (1959). Bacteriophages. New York: Interscience Publishers Inc.

Anderson, E. S. (1951). The significance of Vi-phage types F1 and F2 of Salmonella typhi. J. Hyg., Camb. 49, 158.

Anderson, E. S. (1955). Consideration of the Vi-phage types of Salmonella typhi on a structural basis. Nature, Lond. 175, 171.

ANDERson, E. S. (1956). A new Vi-phage type of Salmonella typhi: with a discussion of methods of preparation of typing phages for new Vi-types. J. gen. Microbiol. 14, 676.

Anderson, E. S. (1959). Mutation in the type-determining phages of Salmonella typhi. Nature, Lond. 184, 1822.

Anderson, E. S. $(1962 a)$. The genetic basis of bacteriophage typing. Brit. med. Bull. 18, 61.

ANDERson, E. S. (1962b). Report of the International Reference Laboratory for enteric phage typing. Ann. Inst. Pasteur, 102, 379.

Anderson, E. S., Armstrong, J. A. \& Niven, J. S. F. (1959). Fluorescence microscopy; observation of virus growth with amino acridines. Symp. Soc. gen. Microbiol. 9, 224.

Anderson, E. S. \& Felix, A. (1952). Variation in Vi-phage II of Salmonella typhi. Nature, Lond. 170, 192.

Anderson, E. S. \& Felix, A. (1953a). Degraded Vi-strains and variation in Vi-phage II of Salmonella typhi. J. gen. Microbiol. 8, 108.

Anderson, E. S. \& Fedix, A. (1953b). The Vi-type determining phages carried by Salmonella typhi. J. gen. Microbiol. 9, 65.

Anderson, E. S. \& Fraser, A. (1955). The influence of the factors determining Vi-type specificity in Salmonella typhi on the adaptation of Vi-phage II. J. gen. Microbiol. 13, 519.

Anderson, E. S. \& Fraser, A. (1956). The statistical distribution of phenotypically modifiable particles and host-range mutants in preparations of Vi-phage II. J. gen. Microbiol. 15, 225.

Anderson, E. S. \& Williams, R. E. O. (1956). Bacteriophage typing of enteric pathogens and staphylococci and its use in epidemiology. J. clin. Path. 9, 91.

Baron, L. S., Formal, S. B. \& Spilman, W. (1953). Use of Vi-phage lysates in genetic transfer. Proc. Soc. exp. Biol., N.Y. 83, 392.

Bernstein, A. (1963). Temperature dependent variation in the Vi-typing reactions of strains of Salmonella typhi. Nature, Lond. 197, 101.

Bertani, G. \& Nice, S. J. (1951). Studies on lysogenesis. II. The effect of temperature on the lysogenization of Shigella dysenteriae with phage P1. J. Bact. 67, 202.

Bertani, G. \& Weigle, J. J. (1953). Host controlled variation in bacterial viruses. $J$. Bact. 65, 113.

Christensen, J. R. (1961). On the process of host-controlled modification of bacteriophage. Virology, 13, 10.

Craigie, J. (1916). The significance and applications of bacteriophage in bacteriological and virus research. Bact. Rev. $10,73$.

Craigie, J. \& Felix, A. (1917). Typing of typhoid bacilli with Vi-bacteriophage. Lancet, i, 823.

Craigie, J. \& YEN, C. E. (1938). The demonstration of types of B. typhosus by means of preparations of type II Vi-phage. Canad. publ. Hlth J. 29, 148, 181.

Drexler, H. \& Christensen, J. R. (1961). Genetic crosses between restricted and unrestricted phage $\mathrm{T} 1$ in lysogenic and nonlysogenic hosts. Virology, 13, 31.

Felix, A. \& Anderson, E. S. (1951). Bacteriophages carried by the Vi-phage types of Salmonella typhi. Nature, Lond. 167, 703. 
Ferguson, W. W., Juenker, A. \& Ferguson, R. A. (1955). Characterization of latent phages from strains of Salmonella typhi typable and untypable with Vi-phage. Amer. J. Hyg. 62, 306.

Fry, B. A. (1959). Conditions for the infection of Escherichia coli with lambda phage and for the establishment of lysogeny. J. gen. Microbiol. 21, 676 .

Jude, A., Nicolle, P. \& Ducrest, P. (1951). Sur la présence simultanée de deux types bactériophagiques (D1 et D6) dans une culture de Salmonella typhi. Ann. Inst. Pasteur, $81,245$.

Lederberg, S. (1957). Suppression of the multiplication of heterologous bacteriophage in lysogenic bacteria. Virology, 3, 496.

LiEB, M. (1953). The establishment of lysogenicity in Escherichia coli. J. Bact. 65, 642.

Luria, S. E. (1953). Host-induced modification of viruses. Cold. Spr. Harb. Symp. quant. Biol. 18, 237.

Luria, S. E. \& Human, M. L. (1952). A non-hereditary, host-induced variation of bacterial viruses. J. Bact. 64, 557.

Nicolle, P., Hamon, Y. \& Diverneau, G. (1962). La lysogénie et la lysogénisation dans leurs rapports avec la lysotypie; Exemples tirées du groupe lysotypique $\mathbf{M}$ de Salmonella typhi. Arch. Roum. Pathol. expér. 21, 315.

Read, K. S. \& Ferguson, W. W. (1961). Control of Vi-phage type of Salmonella typhi by polylysogenicity. Ann. Inst. Pasteur, 100, 120.

Scholtens, R. Th. (1955). Vi-phage typing of Salmonella typhi. Extension of Vi-type group E and observations on group ' 29'. Ann. Inst. Pasteur, 89, 216.

Scholtens, R. TH. (1956). The relationship of Vi types E3 and E4 of Salmonella typhi to Vi types E1 and D1. Antonie v. Leeuwenhoek, 22, 281.

Swanstrom, M. \& Adams, M. H. (1951). Agar layer method for production of high titer phage stocks. Proc. Soc. exp. Biol., N.Y. 78, 372.

Weigle, J. J. \& Bertani, G. (1953). Variations des bactériophages conditionnées par les bactéries hôtes. Ann. Inst. Pasteur, 84, 175. 